\title{
BMJ Open Role of maternal mental health disorders on stillbirth and infant mortality risk: a protocol for a systematic review and meta-analysis
}

\author{
Akilew A. Adane (D , ${ }^{1}$ Helen D. Bailey, ${ }^{1}$ Rhonda Marriott, ${ }^{2}$ Brad M. Farrant, ${ }^{1}$ \\ Scott W. White, ${ }^{3,4}$ Vera A. Morgan, ${ }^{5}$ Carrington CJ. Shepherd ${ }^{1,2}$
}

To cite: Adane AA, Bailey HD, Marriott $\mathrm{R}$, et al. Role of maternal mental health disorders on stillbirth and infant mortality risk: a protocol for a systematic review and meta-analysis. BMJ Open 2020;10:e036280. doi:10.1136/ bmjopen-2019-036280

- Prepublication history and additional material for this paper are available online. To view these files, please visit the journal online (http://dx.doi. org/10.1136/bmjopen-2019036280).

Received 09 December 2019 Revised 13 March 2020 Accepted 10 April 2020

\section{Check for updates}

(c) Author(s) (or their employer(s)) 2020. Re-use permitted under CC BY-NC. No commercial re-use. See rights and permissions. Published by BMJ.

For numbered affiliations see end of article.

\section{Correspondence to}

Dr Akilew A. Adane;

akilew.adane@telethonkids. org.au

\section{ABSTRACT}

Introduction Maternal mental health disorders such as anxiety and depression are major public health concerns. Evidence shows a link between maternal mental health disorders and preterm birth and low birth weight. However, the impacts of maternal mental health disorders on stillbirth and infant mortality have been less investigated and inconsistent findings have been reported. Thus, using the available literature, we plan to examine whether prenatal maternal mental health disorders impact the risk of stillbirth and infant mortality.

Methods and analysis This systematic review and meta-analysis will adhere to Preferred Reporting Items for Systematic Reviews and Meta-Analyses guidelines and will be registered with the International Prospective Register of Systematic Reviews. Systematic searches will be conducted (from database inception to December 2019) in Medline, Embase, PsycINFO and Scopus for studies examining the association of prenatal mental health disorders and stillbirth and infant mortality. The search will be limited to studies published in English language and in humans only, with no restriction on the year of publication. Two independent reviewers will evaluate records and assess the quality of individual studies. The NewcastleOttawa scales and GRADE (Grading of Recommendations, Assessment, Development and Evaluations) approach will be used to assess the methodological quality and bias of the included studies. In addition to a narrative synthesis, a random-effects meta-analysis will be conducted when sufficient data are available. $\left.\right|^{2}$ statistics will be used to assess between-study heterogeneity in the estimated effect size.

Ethics and dissemination As it will be a systematic review and meta-analysis based on previously published evidence, there will be no requirement for ethical approval. Findings will be published in a peer-reviewed journal and will be presented at various conferences.

PROSPERO registration number 159834 .

\section{INTRODUCTION}

Perinatal death (stillbirth and neonatal death) remains a tragedy for many families around the world, including those in high-income countries, but because the risk factors are not fully understood, ${ }^{1-3}$ the rates of perinatal
Strengths and limitations of this study

- To the best of our our knowledge, this systematic review will be the first to comprehensively synthesise and quantify the impact of maternal mental health disorders on stillbirth and infant mortality.

- Databases will be searched without time restrictions and independent evaluation will be employed.

- The methodological quality and the risk of bias of included studies will be evaluated using validated tools.

- The potential limitation of this review could be the heterogeneity of studies in exposure of interest and restriction to studies in English language.

mortality, particularly stillbirth, have declined only slowly. ${ }^{4}$ Prenatal maternal mental health disorders such as anxiety and depression are important public health concerns because of their high prevalence and their links to both short-term and long-term adverse obstetric and child outcomes. ${ }^{5} 6$ Maternal mental health disorders are one of the potential risk factors for stillbirth and infant mortality. ${ }^{7-9}$ Meta-analytical evidence ${ }^{10}{ }^{11}$ has confirmed associations between maternal depression and anxiety and preterm birth and low birth weight, the leading causes of perinatal mortality.

Potential mechanisms linking maternal mental health disorders and perinatal morbidity and mortality may involve altered intrauterine environment and behavioural pathways. Women with mental health disorders are more likely to have poorer health seeking behaviour and are more likely to misuse illicit substances and drugs, which may further affect their health and capacity to manage all aspects of their pregnancy. ${ }^{12} 13$ The intrauterine mechanisms are suggested to operate through disrupted placental functions because of hormonal changes 
(eg, cortisol), inflammatory response and physiological response. ${ }^{14}$ However, the limited extant studies do not provide a consistent picture on the associations between maternal mental health disorders and stillbirth and infant mortality, ${ }^{13}$ mainly because of the small numbers and/ or because they measured different aspects of maternal mental health. Meta-analysis is a valuable approach to resolve inconsistencies across studies and provide high level of evidence, but, to the best of our knowledge, there are no recent systematic reviews and meta-analyses, which comprehensively evaluate the impact of mental health disorders on stillbirth and infant mortality risk. We found only one systematic review, ${ }^{15}$ which evaluated the effect of maternal psychotic disorder on stillbirth risk, but not other common and less severe mental health disorders. The review was not specific to disorders occurring in the prenatal period and the authors acknowledged that all review studies $(n=6)$ had important methodological limitations such as insufficient statistical power and not being population based. Moreover, several papers ${ }^{7-9} 16-19$ have been published in the area after this review. Given the high prevalence of maternal mental health disorders such as anxiety and depression, up-to-date and comprehensive evidence is required to inform policies and practice, and identify research gaps on the associations between prenatal maternal mental health disorders and early life mortality.

\section{Objectives}

This study aims to summarise the available evidence on the associations between prenatal maternal mental health disorders and stillbirth and infant mortality.

\section{METHODS AND ANALYSIS \\ Search strategy}

This systematic review and meta-analysis will adhere to Preferred Reporting Items for Systematic Reviews and Meta-Analyses guidelines. ${ }^{20}$ Systematic searches will be conducted in four databases (Medline, Embase, PsycINFO and Scopus) from their inception to December 2019. This will be limited to studies published in English language and in humans only, with no restriction on the year of publication. Free texts and medical subject headings terms related to maternal mental health disorders and early life mortality (stillbirth and infant mortality), tailored to each database, will be used to identify the relevant literature. The reference lists and citations of the retrieved articles will also be checked manually for additional studies. Authors of individual studies will be contacted for additional information if required. The search strategies and terms for each database are included as an online supplementary appendix A.

\section{Eligibility criteria}

\section{Inclusion criteria}

Original cross-sectional, case control, cohort or intervention studies will be included in the systematic review if they examined the association of any prenatal maternal mental health disorder (occurring prior to or during pregnancy) and stillbirth or infant mortality. We will include stillbirth (death of a fetus at 20 or more weeks of gestation or as defined by individual studies) and infant death (death of a liveborn baby in the first year of life). The association should be presented as odds ratios or relative risks estimates or there should be sufficient information to calculate either of these estimates. In addition, individual studies should include at least one stillbirth/ infant mortality case in both exposed and unexposed groups.

\section{Exclusion criteria}

Correspondence, theses, reviews, editorials, case-only studies and conference abstracts will be excluded. Animalonly studies will also be excluded. If there are multiple publications reporting on the same cohort of women, the most recent and/or the largest by sample size will be included. Studies where it is unclear if the onset of the maternal mental health disorders was prior to birth will be excluded to minimise reverse causality.

\section{Data extraction}

All citations will first be imported into an EndNote library and duplicates will be removed. All records will be screened by their titles. All abstracts that pass the title screening will be uploaded to Rayyan (a systematic review application) and will be reviewed by two independent reviewers (AAA and HDB) based on the inclusion criteria. The full documents of the eligible abstracts will be further examined by the same authors. Finally, the data on the list of authors, country and years of publication, study population, design, exposure and outcome assessment, association/s as well as confounders adjusted for will be extracted into a standardised Excel sheet. The data will also be extracted on other relevant characteristics such as maternal age, infant sex, birth weight and gestational age. At all stages, reviewers involved in the review will have face-to-face meetings to assess and resolve any disagreements on the review. Any unsettled disagreement will be resolved by other members of the research team (CCJS and VAM) who have expertise in the epidemiology of perinatal mental health issues and will not be involved in the independent review process.

\section{Quality and risk of bias assessment}

The Newcastle-Ottawa quality assessment scales ${ }^{21}$ will be used to assess the methodological quality of included studies. The tool assesses three major areas of a case control or cohort study, which includes the selection of the study groups, the comparability of the groups and the ascertainment of either the exposure (for case control studies) or the outcome of interest (for cohort studies). The overall scores range from 0 to 9 , with low (scores between 1 and 4), medium (scores between 5 and 7) or high quality (scores between 8 and 9) grading. The risk of bias for each study will be assessed following the GRADE 
(Grading of Recommendations, Assessment, Development and Evaluations) approach. ${ }^{22}$ Two of the authors (AAA and HDB) will perform the independent quality and risk of bias appraisals.

Funnel plots, a graphical illustration of effect estimates against their measure of precision (eg, SE), will be used to examine the risk of publication bias. In the absence of heterogeneity and bias, studies with high precision are plotted near the top of the funnel, whereas studies with low precision are spread evenly on the bottom sides of the true effect, with the spread narrowing among larger studies with greater precision. Hence, asymmetry in funnel plots in the absence of heterogeneity may suggest possible publication bias, but when a small number of studies are included, it may be hard to differentiate an asymmetric plot occurring because of publication bias from that due to chance. ${ }^{23}$ As a result, an alternative robust meta-analytical technique will be considered to detect and evaluate the risk of publication bias. ${ }^{24}$

\section{Data synthesis}

A systematic narrative synthesis will be conducted to describe the available studies, and when sufficient data are available, random-effects meta-analysis will be conducted for each child outcome (stillbirth, neonatal death and infant mortality) separately and collectively as a composite variable. Accordingly, pooled estimates with $95 \%$ CI will be calculated. ${ }^{25}$ Between-study heterogeneity will be tested using Cochran's $Q$ test and will be further quantified using the index of heterogeneity squared $\left(\mathrm{I}^{2}\right)$ statistic. ${ }^{26}$ The between-study heterogeneity will be considered as low $\left(\mathrm{I}^{2} \leq 25 \%\right)$, moderate $\left(\mathrm{I}^{2} \leq 50 \%\right)$ or substantial $\left(\mathrm{I}^{2} \geq 75 \%\right)$. If there is evidence of significant heterogeneity, the sources of this will be explored through meta-regression and subgroup analyses. Additionally, a range of sensitivity analyses, for example, based on stillbirth definition, maternal mental health disorder definition, types and severity, data collection period (year), design and quality of included studies, will be considered. All the statistical analyses will be performed using STATA V.15 (StataCorp, College Station, Texas, USA).

\section{Patient and public involvement}

Patients and the public will not be involved directly in the design and conduct of the review.

\section{Ethics and dissemination}

As we will only analyse publicly available published data and we will not directly involve individuals, ethical approval is not required for this review. This systematic review protocol will be registered in the International Prospective Register of Systematic Reviews. The findings of this review will be disseminated through publication in a peer-reviewed journal and scientific conferences and meetings. Effort will also be made to circulate findings through newsletters and media releases.

\section{DISCUSSION}

This systematic review and meta-analysis will comprehensively quantify the impacts of prenatal maternal mental health disorders on the risk of stillbirth and infant mortality. The findings will provide important information essential for practitioners and policymakers, identify research gaps in the literature and provide a foundation for future studies in this area.

\section{Strengths and limitations}

To the best of our knowledge, this systematic review will be the first to comprehensively synthesise and quantify the impact of both severe and more common forms of prenatal maternal mental health disorders on stillbirth and infant mortality. The other strengths of this systematic review include the use of several databases with no time restrictions and use of independent screening and evaluation. However, the review is anticipated to have some limitations. These include heterogeneity between studies in the exposure of interest and restriction to studies in English language, which may exclude important literature and compromise generalisability.

\section{Author affiliations}

${ }^{1}$ Telethon Kids Institute, The University of Western Australia, Nedlands, Western Australia, Australia

${ }^{2}$ Ngangk Yira Research Centre for Aboriginal Health \& Social Equity, Murdoch University, Murdoch, Western Australia, Australia

${ }^{3}$ Maternal Fetal Medicine Service, King Edward Memorial Hospital, Subiaco, Western Australia, Australia

${ }^{4}$ Division of Obstetrics and Gynaecology, The University of Western Australia, Nedlands, Western Australia, Australia

${ }^{5}$ Neuropsychiatric Epidemiology Research Unit, School of Population and Global Health, The University of Western Australia, Nedlands, Western Australia, Australia

Contributors AAA, HDB and CCJS conceived the study and developed the search strategy. AAA drafted the protocol and tested the search strategies in consultation with a librarian. RM, BMF, SWW and VAM provided advice on the protocol. All authors critically revised the protocol for methodological and intellectual content and have read and approved the final manuscript.

Funding This research work is supported by funding from an Australian National Health and Medical Research Council (NHMRC) Project Grant (APP1127265), which funds AAA, CCJS and HDB. BMF is funded by an NHRMC Project Grant (APP1098844). The funding bodies had no part in either the study design, conduct, analysis or interpretation of this study.

\section{Competing interests None declared.}

Patient and public involvement Patients and/or the public were not involved in the design, or conduct, or reporting, or dissemination plans of this research.

Patient consent for publication Not required.

Provenance and peer review Not commissioned; externally peer reviewed.

Open access This is an open access article distributed in accordance with the Creative Commons Attribution Non Commercial (CC BY-NC 4.0) license, which permits others to distribute, remix, adapt, build upon this work non-commercially, and license their derivative works on different terms, provided the original work is properly cited, appropriate credit is given, any changes made indicated, and the use is non-commercial. See: http://creativecommons.org/licenses/by-nc/4.0/.

ORCID iD

Akilew A. Adane http://orcid.org/0000-0002-3022-5230 


\section{REFERENCES}

1 Flenady V, Koopmans L, Middleton P, et al. Major risk factors for stillbirth in high-income countries: a systematic review and metaanalysis. Lancet 2011;377:1331-40.

2 Simpson LL. Maternal medical disease: risk of antepartum fetal death. Semin Perinatol 2002;26:42-50.

3 GBD 2015 Child Mortality Collaborators. Global, regional, National, and selected subnational levels of stillbirths, neonatal, infant, and under-5 mortality, 1980-2015: a systematic analysis for the global burden of disease study 2015. Lancet 2016;388:1725-74.

4 Blencowe $\mathrm{H}$, Cousens S, Jassir FB, et al. National, regional, and worldwide estimates of stillbirth rates in 2015, with trends from 2000 : a systematic analysis. Lancet Glob Health 2016;4:e98-108.

5 Bennett HA, Einarson A, Taddio A, et al. Prevalence of depression during pregnancy: systematic review. Obstet Gynecol 2004;103:698-709.

6 Fawcett EJ, Fairbrother N, Cox ML, et al. The prevalence of anxiety disorders during pregnancy and the postpartum period: a multivariate Bayesian meta-analysis. J Clin Psychiatry 2019;80. doi:10.4088/JCP.18r12527. [Epub ahead of print: 23 Jul 2019].

7 Zhong Q-Y, Gelaye B, Fricchione GL, et al. Adverse obstetric and neonatal outcomes complicated by psychosis among pregnant women in the United States. BMC Pregnancy Childbirth 2018;18:120.

8 Ban L, Tata LJ, West J, et al. Live and non-live pregnancy outcomes among women with depression and anxiety: a population-based study. PLoS One 2012;7:e43462.

9 Nilsson E, Hultman CM, Cnattingius S, et al. Schizophrenia and offspring's risk for adverse pregnancy outcomes and infant death. $\mathrm{Br}$ $J$ Psychiatry 2008;193:311-5

10 Grote NK, Bridge JA, Gavin AR, et al. A meta-analysis of depression during pregnancy and the risk of preterm birth, low birth weight, and intrauterine growth restriction. Arch Gen Psychiatry 2010;67:1012-24.

11 Ding X-X, Wu Y-L, Xu S-J, et al. Maternal anxiety during pregnancy and adverse birth outcomes: a systematic review and meta-analysis of prospective cohort studies. J Affect Disord 2014;159:103-10.

12 Zuckerman B, Amaro H, Bauchner H, et al. Depressive symptoms during pregnancy: relationship to poor health behaviors. Am J Obstet Gynecol 1989;160:1107-11.
13 Surkan PJ, Patel SA, Rahman A. Preventing infant and child morbidity and mortality due to maternal depression. Best Pract Res Clin Obstet Gynaecol 2016;36:156-68.

14 Khalifeh $\mathrm{H}$, Brauer R, Toulmin $\mathrm{H}$, et al. Perinatal mental health: what every neonatologist should know. Early Hum Dev 2015;91:649-53.

15 Webb R, Abel K, Pickles A, et al. Mortality in offspring of parents with psychotic disorders: a critical review and meta-analysis. Am J Psychiatry 2005;162:1045-56.

16 Bitew T, Hanlon C, Kebede E, et al. Antenatal depressive symptoms and perinatal complications: a prospective study in rural Ethiopia. BMC Psychiatry 2017;17:301.

17 Chen $\mathrm{Y}-\mathrm{H}$, Tsai S-Y, Lin H-C. Increased mortality risk among offspring of mothers with postnatal depression: a nationwide populationbased study in Taiwan. Psychol Med 2011;41:2287-96.

18 Goedhart G, Snijders AC, Hesselink AE, et al. Maternal depressive symptoms in relation to perinatal mortality and morbidity: results from a large multiethnic cohort study. Psychosom Med 2010;72:769-76.

19 Huang H-C, Sung F-C, Chen P-C, et al. Obstetric outcomes in pregnant women with and without depression: population-based comparison. Sci Rep 2017;7:13937.

20 Moher D, Liberati A, Tetzlaff J, et al. Preferred reporting items for systematic reviews and meta-analyses: the PRISMA statement. PLoS Med 2009;6:e1000097.

21 Wells G. The Newcastle-Ottawa scale (NOS) for assessing the quality of non randomised studies in meta-analyses, 2001. Available: http:// www. ohri. ca/programs/clinical_epidemiology/oxford. asp

22 Higgins JP, Altman DG. Assessing risk of bias in included studies. Cochrane handbook for systematic reviews of interventions: Cochrane book series 2008:187-241.

23 Sterne JAC, Sutton AJ, loannidis JPA, et al. Recommendations for examining and interpreting funnel plot asymmetry in meta-analyses of randomised controlled trials. BMJ 2011;343:d4002.

24 Stanley TD, Doucouliagos H. Meta-Regression approximations to reduce publication selection bias. Res Synth Methods 2014;5:60-78.

25 Harris RJ, Deeks JJ, Altman DG, et al. Metan: Fixed- and RandomEffects meta-analysis. Stata J 2008;8:3-28.

26 Higgins JPT, Thompson SG, Deeks JJ, et al. Measuring inconsistency in meta-analyses. BMJ 2003;327:557-60. 